\title{
100. Epstein-Barr Virus and Human Chromosomes
}

\author{
Growth Characteristics in Culture and Nude Mice of the \\ Virus-Positive, Chromosome 14-Containing \\ Human-Mouse Hybrid Cells ${ }^{\dagger}$
}

\author{
By Kohtaro Yamamoto,*),**) Toyoro Osato,*) \\ Osamu ShIRAToRI,***) Susumu Makino,***) \\ and Ken KATAGIRI***) \\ (Communicated by Sajiro Makino, M. J. A., Oct. 13, 1980)
}

Introduction. The Epstein-Barr virus (EBV) can readily convert normal human lymphocytes into lymphoblasts with infinite replicative capabilities, and is highly suspected of having a causal relationship to human tumors such as Burkitt lymphoma and nasopharyngeal carcinoma (cf. Epstein and Achong, 1979a).

Our previous paper (Yamamoto et al., 1978) has indicated that the resident $\mathrm{EBV}$ genome is to be closely associated with human chromosome 14 and the presence of this particular chromosome alone seems sufficient for the maintenance and the expression of the EBV genetic information in the virus-transformed lymphocytes. It is also documented that changes involving chromosome 14 are highly remarkable in Burkitt tumors (Manolov and Manolova 1972; Jarvis et al., 1974 ; Zech et al., 1976) and in many other lymphoreticular malignancies (cf. Klein, 1979; Epstein and Achong, 1979b).

The present study was then undertaken to inquire into whether or not the close association of EBV genome with chromosome 14 could be related to the mechanism of EBV-induced oncogenesis.

Materials and methods. The human FV5/mouse MCB2 hybrid clones and subclones (Yamamoto et al., 1978) have previously been isolated and characterized in relation to the presence of EBV genome, the expression of the virus-determined nuclear antigen (EBNA) (Reedman and Klein, 1973), and the presence of human chromosomes (Table I). The cells were grown in RPMI 1640 medium supplemented with $10 \%$ heat-inactivated fetal calf serum and $10 \%$ tryptose phos-

t) Address inquiries to: Dr. Toyoro Osato, Department of Virology, Cancer Institute, Hokkaido University School of Medicine, Sapporo 060, Japan.

*) Department of Virology, Cancer Institute, Hokkaido University School of Medicine, Sapporo 060.

**) Present address: Department of Cytogenetics, Medical Research Institute, Tokyo Medical and Dental University, Tokyo 113.

***) Shionogi Research Laboratory, Osaka 660. 
Table I. EBV genome, EBNA, and human chromosomes in FV5/MCB2 hybrid clones and subclones

\begin{tabular}{cccl}
\hline $\begin{array}{l}\text { FV5/MCB2 } \\
\text { clones and subclones }\end{array}$ & EBV genome* & EBNA** & Human chromosomes \\
\hline 7 & $<1$ & 0 & 2,3 \\
10 & $<1$ & 0 & $3,4,5,9,11$ \\
12 & $<1$ & 0 & $13,21,22$ \\
16 & $<1$ & 0 & $1,2,3,15,17,19,20$ \\
17 & $1-2$ & $95-100$ & $2,3,7,14,15,17$ \\
18 & $1-2$ & $95-100$ & $1,2,3,7,14,15,17,21$ \\
19 & $1-2$ & $95-100$ & 14 \\
$19-15$ & $1-2$ & $95-100$ & 14 \\
$19-18$ & $1-2$ & $95-100$ & 14 \\
$*$ Number of EBV genome equivalents per cell. ** Percentage of EBNA- \\
positive cells.
\end{tabular}

phate broth.

The growth characteristics in culture of the hybrid cells were studied referring to the colony-forming efficiency in semisolid agar medium (Mizuno et al., 1976), the maximum saturation density in a given culture dish $(50 \mathrm{~mm})$, and the doubling time in the logarithmic growth phase. The neoplastic growth of the hybrid cells was assessed in $\mathrm{BALB} / \mathrm{c}$ nude mice $(\mathrm{nu} / \mathrm{nu})$, when inoculated subcutaneously.

Results. High growth potential in culture of EBV-positive, chromosome 14-containing FV5/MCB2 hybrid cells. The FV5/MCB2 hybrid clones and subclones containing EBV DNA, EBNA, and human chromosome 14 (positive clones 17, 18, 19 and positive subclones 19-15, 19-18) were compared with the hybrid clones without. containing EBV markers and chromosome 14, but containing some

Table II. Growth in culture of FV5/MCB2 hybrid clones and subclones

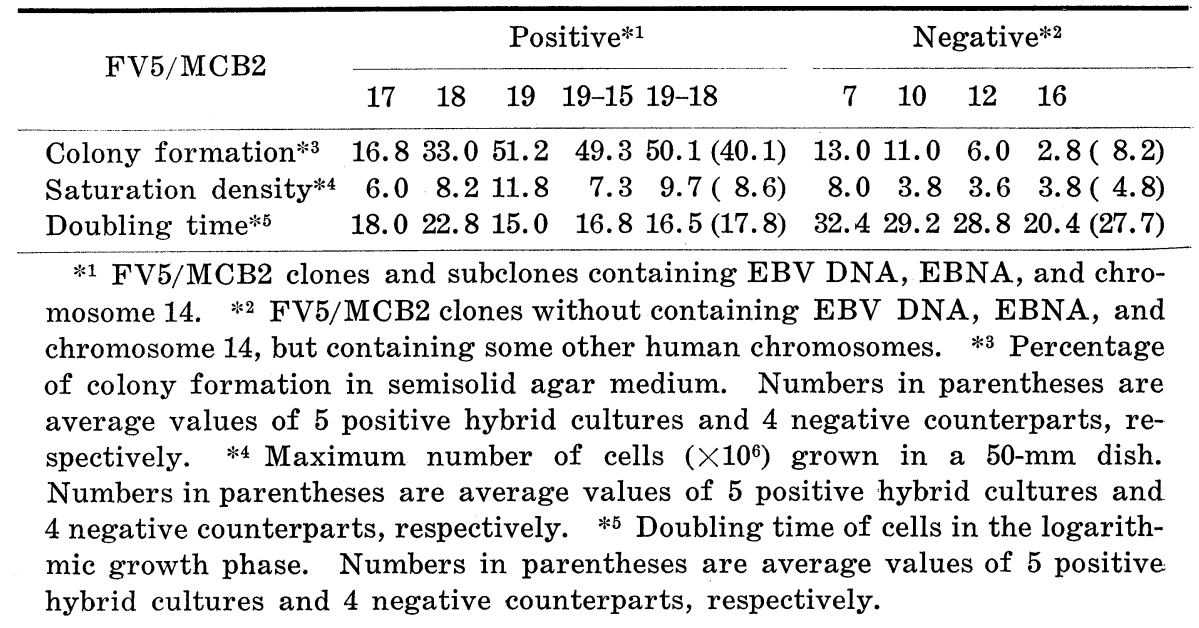


other human chromosomes (negative clones $7,10,12,16)$, with the attention to the mode of growth in culture (Table II). First, when inoculated into semisolid agar medium, the colony-forming efficiency of the 5 positive hybrid cultures was on an average of $40.1 \%$, while the average value was $8.2 \%$ in the 4 negative cultures. Second, the average maximum number of cells per dish from the 5 positive cultures was $8.6 \times 10^{6}$, and $4.8 \times 10^{6}$ in the 4 negative cultures. Third, the average cell doubling time of the 5 positive hybrid cultures was $18.2 \mathrm{~h}$, while the average time in the 4 negative counterparts was $27.7 \mathrm{~h}$.

Neoplastic growth in nude mice of EBV-positive, chromosome 14-containing FV5/MCB2 hybrid cells. Subsequently the above-mentioned 5 EBV DNA-positive, EBNA-expressing, chromosome 14-containing hybrid clones and subclones were assessed for their neoplastic growth capacity in nude mice in parallel condition to the 4 negative clones (Table III). Tumors grew very rapidly by inoculation with a much smaller number of the positive hybrid cells, in comparison with the negative cells.

Table III. Growth in nude mice of FV5/MCB2 hybrid clones and subclones

\begin{tabular}{|c|c|c|c|c|c|c|c|c|c|}
\hline \multirow{2}{*}{ FV5/MCB2 } & \multicolumn{5}{|c|}{ Positive ${ }^{* 1}$} & \multicolumn{4}{|c|}{ Negative*2 } \\
\hline & 17 & 18 & 19 & $19-15$ & $19-18$ & 7 & 10 & 12 & 16 \\
\hline $10^{3 * 3}$ & $0 / 4 * 4$ & $0 / 4$ & $2 / 4$ & $3 / 4$ & $3 / 4$ & $0 / 4$ & $0 / 4$ & $0 / 4$ & $0 / 4$ \\
\hline $10^{4}$ & $3 / 4$ & $4 / 4$ & $4 / 4$ & $4 / 4$ & $4 / 4$ & $0 / 4$ & $0 / 4$ & $0 / 4$ & $0 / 4$ \\
\hline $10^{5}$ & $4 / 4$ & $4 / 4$ & $4 / 4$ & $4 / 4$ & $4 / 4$ & $4 / 4$ & $4 / 4$ & $4 / 4$ & $4 / 4$ \\
\hline
\end{tabular}

*1 FV5/MCB2 clones and subclones containing EBV DNA, EBNA, and chromosome 14. *2 FV5/MCB2 clones without containing EBV DNA, EBNA, and chromosome 14, but containing some other human chromosomes. *3 Number of cells inoculated per mouse. *4 Number of mice developing tumors/ number of mice inoculated.

Discussion. Previously Yamamoto et al. (1978) have assigned the resident EBV genome onto chromosome 14. Also, it has been shown that changes in chromosome 14 are very remarkable in B-cell neoplasias (cf. Klein, 1979; Epstein and Achong, 1979b) including Burkitt tumors (Manolov and Manolova, 1972; Jarvis et al., 1974; Zech et al., 1976). On the above bases the suggestion was possibly made that the close association of EBV genome with chromosome 14 could possibly be related to the mechanism of EBV-induced oncogenesis in human B-lymphocytes (Yamamoto et al., 1978).

Then, data presented in this paper seem to indicate that the resident $\mathrm{EBV}$ genome-positive, EBNA-expressing (i.e. EBNA antigen gene-functioning), human chromosome 14-containing hybrid clones and subclones have a much higher neoplastic growth potential 
as compared with hybrid clones without containing EBV markers and chromosome 14. Growth in culture and in nude mice of the latter negative clones probably reflects growth of the background MCB2 tumor cells which were used as mouse parental cells in our hybridline study (data not given).

Generally speaking, a basic mechanism of EBV-induced oncogenesis in human lymphocytes is possibly explicable on previously proposed views (Yamamoto et al., 1977; Osato, 1979). Upon infection of lymphocytes with EBV, the virus genome, particularly EBNA antigen gene, will be integrated in a specific site of chromosome 14, which may probably be responsible for the growth regulation of B-lymphocytes. Then the integrated EBV genome will impair such regulatory function, and then it results in oncogenesis in EBV-infected lymphocytes. Other mechanisms for the occurrence of Burkitt tumors have also been proposed recently in connection with the possible function of chromosome 14 (Klein, 1979; Epstein and Achong, 1979b).

Acknowledgements. Gratitude is cordially extended to Professor Emeritus Sajiro Makino, M. J. A., for revising this manuscript. We also thank Miss J. Kusajima and Miss T. Izumi for assistance. This work was supported in part by grants from the Ministry of Education, Science and Culture, and the Ministry of Health and Welfare.

\section{References}

Epstein, M. A., and Achong, B. G. (eds.) : The Epstein-Barr Virus. SpringerVerlag, Berlin/Heidelberg/New York (1979a).

Epstein, M. A., and Achong, B. G.: The Epstein-Barr Virus (eds. Epstein, M. A., and Achong, B. G.). Springer-Verlag, Berlin/Heidelberg/New York, p. 321 (1979b).

Jarvis, J. E., Ball, G., Rickinson, A. B., and Epstein, M. A.: Int. J. Cancer, 14, 716 (1974).

Klein, G.: Proc. Nat. Acad. Sci. USA, 76, 2442 (1979).

Manolov, G., and Manolova, Y.: Nature, 237, 33 (1972).

Mizuno, F., Aya, T., and Osato, T.: J. Nat. Cancer Inst., 56, 171 (1976).

Osato, T.: Epstein-Barr Virus Oncogenesis (ed. Osato, T.). Hokkaido University Medical Library Series, Hokkaido University Medical School, Sapporo, vol.11, p. 163 (1979).

Reedman, B. M., and Klein, G.: Int. J. Cancer, 11, 499 (1973).

Yamamoto, K., Izumi, T., Osato, T., Shiratori, O., Makino, S., and Katagiri, K.: Abst. 25th Gen. Meeting Soc. Japan, Virologists, p. 240 (1977).

Yamamoto, K., Mizuno, F., Matsuo, T., Tanaka, A., Nonoyama, M., and Osato, T.: Proc. Nat. Acad. Sci. USA, 75, 5155 (1978).

Zech, L., Haglund, U., Nilsson, K., and Klein, G.: Int. J. Cancer, 17, 47 (1976). 